\title{
Programme scientifique
}

Wissenschaftliches Programm

\section{SORV SSOV \\ HUG \\ Hópitaux Universitaires de Genève \\ DE GENĖVE}

Genève 21-23 septembre 2006/Genf 21.-23. September 2006 Centre Médical Universitaire

\section{Thème principal/Hauptthema: Dermatochirurgie}

Mercredi, 20 septembre 2006/Mittwoch, 20. September 2006

Séance du comité de la SSDV/Sitzung des Vorstandes der SGDV

Jeudi, 21 septembre 2006/Donnerstag, 21. September 2006

Séance du comité de la SSDV/Sitzung des Vorstandes der SGDV

$8.45-16.15 \mathrm{~h}$

Auditoire A250

7e journée de formation continue du Groupe suisse des infirmiers(ères) en dermatologie

7. Fortbildung der Schweizerischen Pflegefachgruppe Dermatologie

$10.30-17.00 \mathrm{~h}$

Auditoire C150

$10.30-10.35 \mathrm{~h}$

$10.35-10.50 \mathrm{~h}$

$10.50-11.10 \mathrm{~h}$

$11.10-11.50 \mathrm{~h}$

$11.50-12.10 \mathrm{~h}$

$12.10-12.45 \mathrm{~h}$

$12.45-13.45 \mathrm{~h}$

$13.45-16.45 \mathrm{~h}$

Salles I, II/IV, V

$18.00-19.30 \mathrm{~h}$

Swissôtel Métropole

$19.30-20.15 \mathrm{~h}$

$20.15 \mathrm{~h}$
Premier cours de formation de dermatochirurgie de la SSDV

Inscription obligatoire

Erster Forbildungskurs für Dermatochirurgie

Anmeldung obligatorisch

Bienvenue/Willkommen

Anatomie chirurgicale/Chirurgische Anatomie

D. Salomon

Fils et techniques de sutures/Nahtmaterial und Technik

M. Adatto

Théorie des lambeaux/Theorie der Lappenplastiken

A. Skaria

Technique de greffe/Technik der Hauttransplantate

J. Hafner

Tips and Tricks in Dermatosurgery

D. Dill-Müller

Snack sandwich - Séance administrative générale

Sandwich-Snack - Generalverwaltungssitzung

Informations Tarmed/Tarmed-Informationen

Atelier pratique «Hands-on training»

J.P. Grillet

Workshop «Hands-on training»

Groupe de travail chirurgie

Arbeitsgruppe für Dermatochirurgie

Cours avancé de chirurgie/Chirurgie für Fortgeschrittene

A Pot-Pourri of Facial Reconstructions: State of the Art

Prof. R. Mellette, University of Colorado, USA

(inscription obligatoire/Anmeldung obligatorisch)

Apéritif/Aperitif

Dîner au Swissôtel Métropole avec l'orateur

Abendessen im Swissôtel Métropole mit dem Redner

(inscription payante obligatoire/Anmeldung obligatorisch, kostenpflichtig) 
Ateliers des groupes de travail de la SSDV

Workshops der Arbeitsgruppen der SGDV

13.00-16.00 h

Salle de microscopie

16.15-17.00 h

Salle de microscopie

$17.15-18.00 \mathrm{~h}$

Auditoire C150

$18.00-18.30 \mathrm{~h}$

Auditoire C150

\section{Dermatopathologie}

Thème: Cancers cutanés non mélanocytaires (NMSC)

Thema: Nichtmelanozytärer Hautkrebs

Observation de lames/Untersuchung der Schnitte

Séance administrative du Groupe de travail dermatopathologie

Verwaltungssitzung der Arbeitsgruppe Dermatopathologie

\section{Dermatopédiatrie}

Cours de dermatochirurgie pédiatrique

Kurs Pädiatrische Dermatochirurgie

Traitement au laser et la chirurgie cutanée chez l'enfant

J. Hohlfeld, Service de chirurgie pédiatrique,

CHUV, Lausanne

Réunion annuelle du Groupe de travail de dermatologie pédiatrique de la SSDV, présentation du nouveau Comité

Jahresversammlung der Arbeitsgruppe Pädiatrische Dermatologie, Vorstellung des neugewählten Vorstandes

(Prof. D. Hohl, I. Izakovic, M. Lacour, A. Lautenschlager, K. Kernland)

Vendredi, 22 septembre 2006/Freitag, 22. September 2006

Dès/ab $8.00 \mathrm{~h}$

$9.00 \mathrm{~h}$

Auditoire B400

9.10-10.00 h

$9.10 \mathrm{~h}$

C1

$9.20 \mathrm{~h}$

C2

$9.30 \mathrm{~h}$

C3

$9.40 \mathrm{~h}$

C4

$9.50 \mathrm{~h}$

C5

$10.00-10.30 \mathrm{~h}$

$10.30-11.00 \mathrm{~h}$
Enregistrement et mise en place des posters (mise en place des posters jusqu'à $10 \mathrm{~h}$ ) Registrierung und Postermontage (Postermontage bis $10 \mathrm{~h}$ )

Ouverture de la Réunion annuelle

Eröffnung der Jahresversammlung

Communications libres

Freie Mitteilungen

Role of Regulatory T Cells in Chronic Graft-versus-Host Disease

P. Häusermann, L. Tabellini, A.C. Damodaran, T.E. Bumgarner, B. Grogan, M.E. Flowers, P.J. Martin, P.A. Carpenter, J.A. Hansen, Universitätsspital Basel

Pemphigus Vulgaris: Antibody Binding to Desmoglein 3 Activates a Signaling Cascade Resulting in Nuclear c-Myc Overexpression in Keratinocytes

L. Williamson, T. Hunziker, M. Suter, E. Müller, University of Berne

Traitement du psoriasis sévère par étanercept: étude sur 16 patients au CHUV

E. Laffitte, M. De Heller, E. Gambillara, A.K. Lapointe, R.G. Panizzon, CHUV, Lausanne

Comparison of Different Surgical Techniques in the Treatment of Pincer Nails

S. Läuchli, J. Hafner, B. Schlagenhauff, R. Baran, Universitätsspital Zürich

L'induction d'une dermite de contact dépend de l'activation de cytokines pro-inflammatoires par l'inflammasome, un senseur des signaux de danger

O. Gaide, H. Watanabe, V. Petrilli, F. Martinon, S. Roques, E. Contassot, J. Tschopp, L.E. French, HUG, Genève

Démonstrations cliniques 1

Klinische Demonstrationen 1

Dermatologische Klinik, Kantonsspital Aarau

Pause, visite des posters et de l'exposition de l'industrie

Pause, Besuch der Poster- und Industrieausstellung 
«So gewährt die plastische Chirurgie, kühn und kräftig gehandhabt, grosse Resultate...» zur Geschichte der Dermatochirurgie

M.L. Geiges, Universitätsspital Zürich

$11.30-12.15 \mathrm{~h}$

$12.15-14.00 \mathrm{~h}$

12.30-13.45 h

Auditoire C150

$12.35 \mathrm{~h}$

$12.50 \mathrm{~h}$

$13.10 \mathrm{~h}$

$13.20 \mathrm{~h}$

Auditoire A250

$14.00-15.00 \mathrm{~h}$

$14.00 \mathrm{~h}$

$14.15 \mathrm{~h}$

$14.45 \mathrm{~h}$

15.00-15.30 h

$15.30-16.30 \mathrm{~h}$

$16.30-17.00 \mathrm{~h}$

$17.00-19.00 \mathrm{~h}$

$20.30 \mathrm{~h}$

Micrographic Surgery from the Basics to the State of Art

Prof. R. Mellette, University of Colorado, USA

Déjeuner, visite des posters et de l'exposition de l'industrie

Mittagessen, Besuch der Poster- und Industrieausstellung

Lunch interactif avec l'industrie (Auditoires C150 et A250)

Interaktiver Lunch mit der Industrie (Hörsäle C150 und A250)

How to Manage the New Treatments in Psoriasis

(sponsorisé par/unterstützt von Serono)

Chairman: Prof. J.-H. Saurat, Hôpital universitaire de Genève

Comorbidity and Psoriasis

Prof. M. Stähle, Karolinska Institutet, Stockholm, Sweden

Raptiva Patient Management

N. Selenko-Gebauer, Medizinische Universität Wien, Österreich

Case Report on Raptiva

A.M. Thielen, Hôpital universitaire de Genève

Question and Answer Session

Cicatrisation - Nouvelles découvertes de la recherche et indications en dermatologie

Wundheilung - Neue Erkenntnisse aus der Forschung und Einsatzbereiche in der Praxis

(sponsorisé par/unterstützt von La Roche-Posay)

D. Beer, ETHZ Zellbiologie, B. Schlagenhauff, Dermacenter Küssnacht,

A. Skaria, Président du Groupe de travail dermatochirurgie,

Recherche La Roche-Posay/L'Oréal

Mini-conférences

Mini-Vorträge

Hémostase et intervention chirurgicale: attitude pratique

Prof. P. de Moerloose, Unité d'hémostase, HUG, Genève

Einsatz und Wertigkeit der Ultraschalldiagnostik in der Dermatologie

D. Dill-Müller, Universitätsklinikum des Saarlandes, Homburg, Deutschland

Technique de comblement par tissu adipeux autologue en dermatologie

A. Skaria, Centre de dermatochirurgie, Vevey, Suisse

Démonstrations cliniques 2

Klinische Demonstrationen 2

Dermatologische Klinik, Universitätsspital Basel

Séminaire de politique professionnelle/Berufspolitisches Seminar

Bedeutung der Aufklärungspflicht von Patienten

Arzt und Strafrecht

G. Sasse, Kantonsspital Aarau

Pause, visite des posters et de l'exposition de l'industrie

Pause, Besuch der Poster- und Industrieausstellung

Assemblée générale de la SSDV

Generalversammlung der SGDV

Banquet avec remise des prix/Bankett mit Preisverleihungen

Restaurant Parc des Eaux-Vives 
$8.00-13.30 \mathrm{~h}$

8.30-9.00 h

Auditoire B400

$9.00-9.30 \mathrm{~h}$

$9.30-10.15 \mathrm{~h}$

$10.15-10.45 \mathrm{~h}$

$10.45-11.15 \mathrm{~h}$

$11.15-11.45 \mathrm{~h}$

$11.45-12.15 \mathrm{~h}$

$12.15-14.00 \mathrm{~h}$

$12.30-13.45 \mathrm{~h}$

Auditoire $\mathrm{C} 150$

Auditoire A250

$14.00-14.30 \mathrm{~h}$

$14.30-15.00 \mathrm{~h}$

$15.30 \mathrm{~h}$
Enregistrement/Registrierung

Démonstrations cliniques 3

Klinische Demonstrationen 3

Dermatologische Universitätsklinik, Inselspital, Bern

Démonstrations cliniques 4

Klinische Demonstrationen 4

Service de dermatologie, CHUV, Lausanne

Correlations histopathologiques et cliniques, discussion des cas de dermatopathologie

Histopathologisch-klinische Korrelationen, Besprechung der Fälle der Dermatopathologie

Pause, visite des posters et de l'exposition de l'industrie

Pause, Besuch der Poster- und Industrieausstellung

Démonstrations cliniques 5

Klinische Demonstrationen 5

Dermatologische Klinik, Universitätsspital Zürich

Pearls in Dermatological Surgery

Prof. E. Haneke, Inselspital, Bern

Les dermatologues et les risques anesthésiques

Dr P. Mathelier-Fusade, Hôpital Tenon, Paris, France

Lunch, visite des posters et de l'exposition de l'industrie

Lunch, Besuch der Poster- und Industrieausstellung

Lunch interactif avec l'industrie (Auditoires C150 et A250)

Interaktiver Lunch mit der Industrie (Hörsäle C150 und A250)

Aktuelles zur Aknetherapie 2006

(sponsorisé par/unterstützt von Galderma)

M. Harms, Genève, Suisse, E. Delaporte, Lille, France, M. Kägi, Zürich, Schweiz

Chute de cheveux et dermocosmétiques: de la constatation aux solutions

Haarausfall und Dermokosmetika: von der Vision zur Praxis

(sponsorisé par/unterstützt von Vichy)

Prof. R. Trüeb, Universitätsspital Zürich

Démonstrations cliniques 6

Klinische Demonstrationen 6

Dermatologisches Ambulatorium, Stadtspital Triemli, Zürich

Démonstrations cliniques 7

Klinische Demonstrationen 7

Service de dermatologie, HUG, Genève

Clôture de la Réunion annuelle

Ende der Jahresversammlung 


\section{7e journée de formation continue du Groupe suisse des infirmiers(ères) en dermatologie, le 21 septembre 2006 \\ 7. Fortbildung der Schweizerischen Pflegefachgruppe Dermatologie am 21. September 2006}

Le service de dermatologie de Genève a le plaisir d'organiser la 7e journée de formation continue avec la participation du Groupe suisse des infirmiers(ères) en dermatologie.

Le Comité d'organisation et les Responsables de dermatologie des Hôpitaux universitaires de Genève se réjouissent de vous accueillir.

Cette journée se déroulera en parallèle du congrès annuel de la SSDV, avec le soutien du Professeur Saurat et la participation de Johnson et Johnson.

\section{Déroulement de la journée de congrès}

Ouverture

Accueil et introduction par un membre de la direction des soins infirmiers des HUG

Présentation de l'Association SPFD: Mme R. De Lorenzo

Modérateur du jour: Mme. L. Blal

Le psoriasis, simple problème esthétique ou maladie invalidante?

Mme S. Von der Weid et Mme. B. Jolliet, Infirmières, HUG, Genève

\section{Pause café}

Psoriasis - Die Anwendung von topischen Therapien im Tuch Herr M. Musholt und Frau S. Kruse, Pflegefachpersonen, Kantonsspital Zürich

Die Göckermanntherapie

Frau B. Zurbuchen und Frau E. Sane, Pflegefachfrauen, Inselspital Bern

Apéritif et pause repas

Technique d'antalgie «Méopa»

Dr Lucian Macréa, HUG, Genève

Utilisation du Méopa en dermatologie: de l'enseignement qualifiant à l'autonomie, le rôle de l'infirmière référente équipe antalgie

Mme A. Vinsonneau, Infirmière spécialiste clinique équipe antalgie, HUG, Genève

Le Méopa en dermatologie, déroulement du soin et expériences de soignants Mme M.J. Kouevi et M. G. Labatut, Infirmiers HUG, Genève

Clôture par Mme. R. de Lorenzo, Présidente du Groupe suisse des infirmières en dermatologie

\section{Pour tous renseignements}

Mme Rosaria De Lorenzo, Présidente de l'Association suisse de dermatologie

rosaria.delorenzo@ksa.ch

Tél. 0628386952

M. Dominique Conan, Infirmier responsable secteurs ambulatoires de dermatologie

dominique.conan.@hcugech

Tél. 02237 29451, bip 6853451

Mme Caroline Millet, infirmière responsable de dermatologie

millet-caroline@diogenes.hcuge.ch

Tél. 0223753450 , bip 6857784 